\title{
Histology and Clinical Characteristics of Asymptomatic Treatment-naïve Children with Chronic Hepatitis B in Shanghai China
}

\section{Yao Hu ( $\square$ yaohu19890123@126.com )}

Children's Hospital of Fudan University https://orcid.org/0000-0001-5175-6164

\section{Xia Wu}

Children's Hospital of Fudan University

\section{Yingzi Ye}

Children's Hospital of Fudan University

\section{Lijing Ye}

Children's Hospital of Fudan University

\section{Shuzhen Han}

Children's Hospital of Fudan University

\section{Qirong Zhu}

Children's Hospital of Fudan University

\section{Xiaohong Wang}

Children's Hospital of Fudan University

Hui Yu

Children's Hospital of Fudan University

\section{Research Article}

Keywords: Children, Asymptomatic, Treatment-naïve, Chronic Hepatitis B, Histology, Pathology, Inflammation, Fibrosis, Cirrhosis, Clinical Characteristics

Posted Date: April 20th, 2021

DOl: https://doi.org/10.21203/rs.3.rs-414418/v1

License: (c) (1) This work is licensed under a Creative Commons Attribution 4.0 International License. Read Full License 


\section{Abstract}

BACKGROUND \& AIMS: Chronic hepatitis B (CHB) virus infection is a major cause of morbidity and mortality. We aim to investigate the association between hepatic histology and clinical characteristics in asymptomatic treatment-naïve children chronically infected with hepatitis B virus (HBV) in Shanghai China.

METHODS: Liver biopsies of 278 asymptomatic treatment-naïve children with chronic hepatitis B virus infection were scored for inflammation and fibrosis, and correlated with clinical and laboratory data.

RESULTS: The clinical, virologic and pathologic features of chronic HBV infection were studied in 278 treatment-naïve children from Shanghai, China, of who were all asymptomatic. And $177(63.7 \%)$ of the children were male. Sera from mothers of 277 of these children were HBsAg positive. Up to $87.4 \%$ of subjects were hepatitis B e antigen-positive at the time of the biopsy. The median age at biopsy was 5.1 years (interquartile range 2.8-8.4 years). HBV DNA levels were generally high (mean $7.4 \log _{10} \mathrm{IU} / \mathrm{mL}$ ), as was serum alanine aminotransferase (median $105 \mathrm{U} / \mathrm{L}$ ). Using the Ishak-modified histology activity index scoring system, no inflammation was seen in $2.9 \%$ cases, mild inflammation was in $22.3 \%$, moderate in $73.4 \%$, and severe in $1.4 \%$. No fibrosis was seen in $11.5 \%$, mild fibrosis in $32.7 \%$, moderate fibrosis in $47.5 \%$, and cirrhosis in $8.3 \%$. When serum ALT level was $\leq 80(2 \times U L N)$ and $>80 \mathrm{U} / \mathrm{L}$, the degree of inflammation $(P<0.0001)$ and fibrosis stage $(P<0.0001)$ had significant differences. Inflammation and fibrosis aggravated with ALT level increasing. Fibrosis stage had significant differences between age of $\leq 3$ and $>3$ years $(P<0.0001)$. Moderate fibrosis and cirrhosis rate was relatively higher in children aged $\leq 3$ years at biopsy. No correlation was found between histological changes with gender, HBV genotypes or HBV DNA levels.

CONCLUSION: Significantly wide range of inflammation and fibrosis is seen in asymptomatic treatmentnaïve children with chronic hepatitis B in Shanghai China. Serum ALT >80 U/L may be a strong indicator of the degree of hepatic inflammation and severity of fibrosis. Moderate fibrosis and cirrhosis has already appeared in aged $\leq 3$ years children.

\section{Introduction}

Hepatitis B virus (HBV) infection is a major public health concern worldwide. Chronic HBV infection is extremely harmful and is closely associated with the development of cirrhosis, hepatocellular carcinoma (HCC), and liver failure [1]. Infection with HBV at a young age is known to lead frequently to the chronic hepatitis B surface antigen ( $\mathrm{HBsAg}$ ) carrier state, particularly if it occurs during the perinatal period. More than half of existing chronic hepatitis $B(\mathrm{CHB})$ patients were estimatedly infected in perinatal period in China [2-4]. All of these facts highlight the importance of chronic HBV infection in early childhood for the eventual development of serious chronic liver disease.

As we all known, liver biopsy is the gold standard in the histological diagnosis and management of patients with chronic hepatitis B. Liver biopsy can help to determine the hepatic inflammation degree and 
fibrosis stage and to identify other co-existent liver conditions that may influence disease progression [5]. Several studies have revealed variable severity of disease in chronic HBV infection in children [6-9].

Chronically HBV infection is a dynamic process, which is influenced by many factors, such as host immune response, age, duration of disease, viral load, and HBV genotype $[5,10]$. It was revealed that chronically HBV infection patients with elevated HBV DNA levels and serum alanine aminotransferase (ALT) levels are at higher risk of developing complications in adults [11].

This study was conducted to correlate the histopathological changes with demographic, clinical, and virologic characteristics at the time of the liver biopsy for asymptomatic treatment-naïve children with chronic hepatitis B from Shanghai, China.

\section{Patients And Methods}

\section{Included and exclusive criteria}

Included criteria: (1) Patients aged < 18 years were eligible if they were HBsAg-positive or HBV DNA positive. (2) All of the subjects were asymptomatic and treatment-naïve. (3) All children had no history of blood products transfusion before biopsy. (4) All the children were willing to provide informed consent.

Exclusive criteria: (1) Patients were ineligible if they had tested positive for hepatitis A, C, D or E or for HIV, or had a history or evidence of chronic liver disease other than CHB, or suspicion of HCC. (2) Patients with a history of significant chronic pulmonary or cardiac disease, renal disease, thyroid disease or diabetes, immunodeficiency disease, previous solid organ or stem-cell transplant, or evidence or history of malignancy were also excluded. (3) Other definitive liver diseases, including autoimmune hepatitis, metabolic liver disease, fatty liver disease and so on, were eliminated.

The study was approved by the Ethics Committee of the Children's Hospital of Fudan University, and written informed consent was obtained from every child's parent(s).

\section{Subjects and Specimens}

All subjects participating in the study underwent extensive evaluation including detailed history, physical, and laboratory examinations, of whom visited between January, 2002 and December, 2020. Clinical and demographical information including sex, age at time of biopsy, mode of transmission, HBV genotype, ALT level, HBV DNA level, HBsAg, and hepatitis B envelope antigen (HBeAg) were collected.

Liver biopsies obtained from 286 chronic HBsAg carrier children were collected. Two of them were excluded as patients were previously treated for hepatitis B. One biopsy was excluded from the group because of the teratoma and chemotherapy history. One was excluded for with Wilson's disease. And four were excluded for data integrity. And 278 liver biopsy specimens were used for the final analysis.

\section{Histologic Studies}


All specimens were stained with hematoxylin-eosin and Masson's trichrome and scored for inflammation and fibrosis using the Ishak [12] and Metavir [13] scoring systems. All biopsies were de-identified and coded and examined by a single expert histopathologist.

To demonstrate liver HBsAg and hepatitis B core antigen ( $\mathrm{HBcAg}$ ), the deparaffinized sections were stained by the peroxidase-antiperoxidase (PAP) technique [14] using mouse monoclonal anti-HBs and rabbit anti-HBc (Sigma) [15].

\section{Statistics}

Associations between continuous functions and histological data, inflammation and fibrosis, were assessed using Spearman correlation. All $P$ values were 2-sided, with $P<0.05$ considered statistically significant. Kruskal-Wallis test or Mann-Whitney test was used for statistical analysis of differences between uncontinuous variable groups. SPSS (version 22) and GraphPad Prism (version 7) were used for all analysis and graphing.

\section{Results}

\section{Patient Characteristics}

Of the 278 subjects whose liver biopsies and clinical characteristics were reviewed, all of them were asymptomatic and treatment-naïve. And $63.7 \%$ (177/278) of them were male (Table 1). The maternal HBsAg-positive rate was up to $99.6 \%$ (277/278), excepted one was adopted by Welfare homes, based on the information obtained from detailed history. And no one had a history of blood products transfusion before biopsy. 
Table 1

Demographics and Baseline Disease characteristics

\section{Frequency $(\%)$ or median [25th to 75 th percentile]}

\begin{tabular}{ll} 
Age at biopsy, years & $5.1[2.8-8.4]$ \\
Sex, male & $177(63.7)$ \\
\hline HBeAg status & \\
Positive & $243(87.4)$ \\
\hline Negative & $35(12.6)$ \\
\hline ALT, U/L & $105[65-175]$ \\
\hline HBV DNA, $\log _{10} \mathrm{IU} / \mathrm{mL}$ & $7.4[6.8-8.0]$ \\
\hline Detectable & $269(96.8)$ \\
\hline Undetectable & $9(3.2)$
\end{tabular}

HBV genotypes, $n=132$

$\begin{array}{ll}\text { B } & 43(32.6) \\ \text { C } & 89(67.4)\end{array}$

The normal range for ALT used in this study was 0-40 U/L for both male and female individuals. Up to $87.4 \%$ of the subjects were HBeAg positive, $12.6 \%$ were HBeAg-negative at the time of biopsy. All children were HBsAg-positive (100\%). Among 132 subjects tested for HBV genotype, the HBV genotype distribution was narrow (B: $32.6 \%$; C: $67.4 \%$; no other genotypes).

The median age at biopsy was 5.1 years, ranged from 8-month to 15-year-3-month (interquartile range 2.8-8.4 years). Sixty-nine $(24.8 \%, 69 / 278)$ of them aged under 3 years old. At the time of the biopsy, 269 subjects were HBV DNA positive, and 9 subjects were HBV DNA undetectable $(<1000 \mathrm{IU} / \mathrm{mL}$ before 2019 , $<500 \mathrm{IU} / \mathrm{mL}$ since 2019). Mean HBV DNA levels of the 269 detectable subjects were $7.4 \log _{10} \mathrm{IU} / \mathrm{mL}$ (range 3.1-9.9 $\log _{10} \mathrm{IU} / \mathrm{mL}$ ). Serum ALT ranged from 5 to $2170 \mathrm{U} / \mathrm{L}$ (median 105, interquartile range 65175).

\section{Histological Findings}

The degree of inflammation seen in the liver biopsies ranged from none to severe inflammation whereas the presence of fibrosis stage ranged from none to cirrhosis (Fig. 1). Using the Ishak modified histology activity index scoring system, no inflammation was seen in $2.9 \%$ (score $0,8 / 278$ ), mild inflammation was seen in 22.3\% (score 1, 62/278), moderate inflammation in 73.4\% (score 2, 48.6\%, 135/278; score 3 , $24.8 \%, 69 / 278$ ), and severe inflammation in $1.4 \%$ (score $4,4 / 278$ ). 
No fibrosis was seen in $11.5 \%$ (score $0,32 / 278$ ), mild fibrosis in $32.7 \%$ (score 1,91/278), moderate fibrosis (including advanced fibrosis consisting of bridging fibrosis) was present in $47.5 \%$ (score $2,37.4 \%$, $104 / 278$; score $3,10.1 \%, 28 / 278)$, and cirrhosis in $8.3 \%(23 / 278)$.

Liver HBsAg was detected in 278 cases. HBsAg immunostaining positive rate was in $92.1 \%(256 / 278)$ of biopsies. HBcAg was detected in 276 of the cases in liver. And HBcAg immunostaining positive rate was $30.8 \%(85 / 276)$.

\section{Association between Clinical Characteristics and Pathologic Histology}

It was found that ALT level was moderately associated with hepatic inflammation degree $(r=0.39, P<$ $0.0001)$ and fibrosis stage $(r=0.38, P<0.0001)$. And when the serum ALT level was less than or equal to $80(\leq 80)$ and higher than $80(>80) \mathrm{U} / \mathrm{L}$ (2 times of upper normal limitation), the degree of inflammation $(P<0.0001)$ and fibrosis stage $(P<0.0001)$ had significant differences (Fig. 2). And the percentage of moderate fibrosis and cirrhosis in ALT $\leq 80 \mathrm{U} / \mathrm{L}$ and ALT $>80 \mathrm{U} / \mathrm{L}$ children was $39.1 \%(50 / 128)$ and $70.0 \%$ $(105 / 150)(P<0.0001)$, respectively.

There was no association between inflammation degree and age at biopsy $(P=0.46)$. But for the association between fibrosis and age at biopsy, our correlation coefficient was $-0.19, P=0.0018$. And it was found that fibrosis stage had significant differences between less than or equal to the age of $3(\leq 3)$ and older than $3(>3)$ years $(P<0.0001)$ (Fig. 3).

The percentage of elevated ALT higher than $80 \mathrm{U} / \mathrm{L}$ in children aged $\leq 3$ years at biopsy was $66.7 \%$ $(46 / 69)$, while that was $49.8 \%$ (104/209) in children older than 3 years at biopsy. And the percentage of moderate fibrosis and cirrhosis in age of $\leq 3$ and $>3$ years children with ALT $>80 \mathrm{U} / \mathrm{L}$ was $80.4 \%(37 / 46)$ and $65.4 \%(68 / 104)$, respectively.

No associations were found between inflammation degree and gender, HBV DNA levels, or HBV genotypes (all $P$ values $>0.05$ ). And no associations were found between fibrosis stage and gender, $\mathrm{HBV}$ DNA levels, or HBV genotypes (all $P$ values $>0.05$ ).

\section{Discussion}

This study of 278 asymptomatic treatment-naïve children with chronic hepatitis B infection from China revealed a wide range in severity of liver disease. Moderate inflammation was seen in a large number of the children chronically infected with HBV from Shanghai. Most of maternal HBsAg was positive (99.6\%), and no one had a history of blood products transfusion before biopsy. Although most of the children visited at the age of more than 1 year old, we still have reason to believe that mother-to-child vertical transmission is the main route of transmission. And most of the subjects were HBeAg positive ( $87 \%)$.

Higher serum ALT level indicates higher liver inflammation degree and fibrosis stage in children with chronic hepatitis B, consisting with the previous reports [6-9]. In our study, we found that when the serum 
ALT level was higher than 2 times of upper normal limitation $(80 \mathrm{U} / \mathrm{L})$, the degree of inflammation and fibrosis stage was found to be relatively severe. Fibrosis was present in most specimens $(88.5 \%$, 246/278) and also correlated with serum ALT levels. And moderate fibrosis and cirrhosis rate in ALT $\leq 80$ $\mathrm{U} / \mathrm{L}$ and $\mathrm{ALT}>80 \mathrm{U} / \mathrm{L}$ children was $39.1 \%$ vs $70.0 \%(P<0.0001)$. It is important to recognize that biopsy should perform as soon as pediatrician can, even though ALT elevation might be mild, which is generally accepted immunetolerant phase. So providing close monitoring of pediatric patients is recommended by more and more pediatricians $[8,9,16,17]$.

Cirrhosis does occasionally develop during childhood $[9,18]$, despite it is generally believed to be rare in children with chronic HBV previously. It was revealed that cirrhosis was found in $3 \%$ patients in one study of 292 children with HBV infection and elevated serum ALT levels [19]. But a higher HDV co-infection rate was reported in these cirrhosis children compared with those without cirrhosis, which is generally believed may have contributed to disease progression. In our current study, 23 children had cirrhosis (8.3\%), 14 of them below the age of 6 years. And 2 of the 14 cases were being 8 months and 9 months old at the time of biopsy. And none of them had HDV or HCV co-infection. One study of $76 \mathrm{HBeAg}$-positive children with chronic hepatitis B and elevated serum ALT levels, moderate-to-severe fibrosis was reported in at least $50 \%$ children of them [18]. In our study, most of the subjects had different degree of fibrosis, and moderate-to-severe fibrosis (cirrhosis) rate was up to $55.8 \%$. And all these findings suggest that chronic HBV infection in children is at risk for developing cirrhosis, which highlights the importance of close monitoring of pediatric patients with $\mathrm{CHB}$ once again.

Contrary to the results seen in adults in which study have shown an association of higher extent of inflammation degree and advanced fibrosis with age over 40 years and duration of disease [20]. The median age at biopsy was 5.1 years, ranged from 8-month to 15-year-3-month (interquartile range 2.88.4 years) in our this study. Sixty-nine (24.8\%) of them aged under 3 years old. There was not significant correlation between the inflammation degree and age at biopsy. But we found that fibrosis stage had significant differences between age of $\leq 3$ and $>3$ years children $(P<0.0001)$. And the percentage of moderate fibrosis and cirrhosis in aged $\leq 3$ years and $>3$ years children with ALT $>80 \mathrm{U} / \mathrm{L}$ was $80.4 \%$ and $65.4 \%(P<0.0001)$. One of the limitations of this study is that we did not analyzed the influence of duration of disease on liver pathology, for the uncertainty of exactly time of HBV infection in some children.

In accord with the male predominance in $\mathrm{HCC}[3,21-24]$, a greater male predominance was demonstrated in children with CHB in our this study (63.7\%). But no associations were found between inflammation degree or fibrosis stage and male sex, which may because the duration of infection was not long enough. For that, we need bigger sample size and long-time follow-up study.

No associations were found between inflammation degree $(P>0.05)$ or fibrosis stage $(P>0.05)$ and HBV DNA levels in this study, in according with some previous studies $[8,9]$. There is a wide distribution of HBV genotypes in countries of North America $[8,22,25,26]$. In one of the largest studies, 343 children and adolescents aged $1-18$ years with chronic hepatitis $B, H B V$ genotype $B$ or $C$ is dominant $(43 \%$ and $32 \%$, 
respectively) [25]. The others had genotype A (5\%), D (16\%), or E (4\%). HBV genotypes B and C are predominant in Asia, and our result is similar to the genotype distribution in these studies [4, 23, 27]. No correlation was identified between genotype and degree of inflammation or fibrosis. And one of the limitations of our study is that we did not establish a association between the presence of other HBV genotypes and liver histology, as we only had genotypes B $(32.6 \%, 43 / 132)$ and C $(67.6 \%, 89 / 132)$. A long-term detailed combined clinical, virologic, immunohistologic and pathological study may provide more valuable insights into the natural course of HBV infection in children.

In conclusion, the results of this study demonstrate that significantly wide range of inflammation and fibrosis is seen in asymptomatic treatment-naïve children with chronically hepatitis B virus infection in Shanghai China. Serum ALT > $80 \mathrm{U} / \mathrm{L}$ may be a strong indicator of the degree of hepatic inflammation and severity of fibrosis. Moderate fibrosis and cirrhosis has already appeared in aged $\leq 3$ years children. And it is very important to monitor pediatric patients with chronic hepatitis $\mathrm{B}$ closely in clinical practice.

\section{Abbreviations}

$\mathrm{HCC}$, hepatocellular carcinoma; $\mathrm{CHB}$, chronic hepatitis B; HBeAg, hepatitis B e antigen; HBsAg, hepatitis B surface antigen; $H B C A g$, hepatitis $B$ core antigen; HBV, hepatitis $B$ virus; ALT, alanine aminotransferase; ULN, upper limit of normal.

\section{Declarations}

Author contributions: Hui Yu designed the study, with input from Qirong Zhu and Xiaohong Wang. Yao Hu and Xia Wu analyzed and, together with Yingzi Ye, Lijing Ye, and Shuzhen Han, interpreted the data. Yao Hu drafted, and all authors critically revised, the manuscript.

Conflict of Interest: All the authors have nothing to disclose.

Data Availability: All of the data are available to all the readers.

Animal Research (Ethics): Not applicable.

Consent to Participate (Ethics): Written informed consent is obtained from every child's parent(s).

Consent to Publish (Ethics): The study was approved by the Ethics Committee of the Hospital.

Plant Reproducibility: Not applicable.

Clinical Trials Registration: Not applicable.

Funding: No funding.

\section{Acknowledgments}

The authors would like to acknowledge all the staff who helped with and supported this study, especially histopathologist Pro. Xiqi Hu and all his staff. The authors also acknowledge all the participating children and their families. 


\section{References}

1. Lozano R, Naghavi M, Foreman K, et al. Global and regional mortality from 235 causes of death for 20 age groups in 1990 and 2010: a systematic analysis for the Global Burden of Disease Study 2010. Lancet. 2012;380:2095-128.

2. Wang FS, Fan JG, Zhang Z, et al. The global burden of liver disease: the major impact of China. Hepatology. 2014;60:2099-108.

3. Hou J, Wang G, Wang F, et al. Guideline of Prevention and Treatment for Chronic Hepatitis B (2015 Update). J Clin TransI Hepatol. 2017;5:297-318.

4. Hou J, Cui F, Ding Y, et al. Management Algorithm for Interrupting Mother-to-Child Transmission of Hepatitis B Virus. Clin Gastroenterol Hepatol. 2019;17:1929-36.

5. Das P, Ahuja A, Gupta SD. Overview of the histopathology of chronic hepatitis B infection. Hepatitis B Annual. 2012;9:49-85.

6. Hsu HC, Lin $\mathrm{YH}$, Chang MH, et al. Pathology of chronic hepatitis B virus infection in children: with special reference to the intrahepatic expression of hepatitis B virus antigens. Hepatology. 1988;8:378-82.

7. Iorio R, Giannattasio A, Cirillo F, et al. Long-term outcome in children with chronic hepatitis B: a 24year observation period. Clin Infect Dis. 2007;45:943-9.

8. Rodriguez-Baez N, Murray KF, Kleiner DE, et al. Hepatic Histology in Treatment-naïve Children With Chronic Hepatitis B Infection Living in the United States and Canada. J Pediatr Gastroenterol Nutr. 2020;71:99-105.

9. Zhang HF, Yang XJ, Zhu SS, et al. Pathological changes and clinical manifestations of 1020 children with liver diseases confirmed by biopsy. Hepatobiliary Pancreat Dis Int. 2004;3:395-8.

10. Mani H, Kleiner DE. Liver Biopsy Findings in Chronic Hepatitis B. Hepatology. 2009;49S:61-71.

11. Perz JF, Armstrong GL, Farrington LA, et al. The contributions of hepatitis $B$ virus and hepatitis $C$ virus infections to cirrhosis and primary liver cancer worldwide. J Hepatol. 2006;45:529-38.

12. Ishak K, Baptista A, Bianchi L, et al. Histological grading and staging of chronic hepatitis. J Hepatol. 1995;22(6):696-9.

13. Bedossa P, Poynard T. An algorithm for the grading of activity in chronic hepatitis C. Hepatology. 1996;24(2):289-93.

14. Su IJ, Lai MY, Hsu HC, et al. Diverse virological, histopathological and prognostic implications of seroconversion from hepatitis $B$ e antigen to anti-HBe in chronic hepatitis B virus infection. $J$ Hepatol. 1986;3:182-9.

15. Chang $\mathrm{MH}$, Hsu HC, Lee $\mathrm{CY}$, et al. Fraternal hepatocellular-carcinoma in young-children in 2 families. Cancer. 1984;53:1807-10.

16. Zhu S, Zhang H, Dong Y, et al. Antiviral therapy in hepatitis B virus-infected children with immunetolerant characteristics: A pilot open-label randomized study. J Hepatol. 2018;68:1123-8. 
17. Wu JF, Song SH, Lee CS, et al. Clinical Predictors of Liver Fibrosis in Patients With Chronic Hepatitis B Virus Infection From Children to Adults. J Infect Dis. 2018;217:1408-16.

18. Godra A, Perez-Atayde A, Jonas MM. Histologic features of chronic hepatitis B in children. Hepatology. 2005;421:478A-479A.

19. Bortolotti F, Calzia R, Cadrobbi P, et al. Liver-cirrhosis associated with chronic hepatitis B virus infection in childhood. J Pediatr. 1986;108:224-7.

20. Cadranel JF, Lahmek P, Causse $X$, et al. Epidemiology of chronic hepatitis B infection in France: risk factors for significant fibrosis - results of a nationwide survey. Aliment Pharmacol Ther. 2007;26:565-76.

21. Terrault NA, Lok AS, McMahon BJ, et al. Update on Prevention, Diagnosis, and Treatment and of Chronic Hepatitis B: AASLD 2018 Hepatitis B Guidance. Hepatology. 2018;67:1560-99.

22. EASL. EASL 2017 Clinical Practice Guidelines on the management of hepatitis B virus infection. J Hepatol 2017;67:370-398.

23. Vittal A, Ghany MG. WHO Guidelines for Prevention, Care and Treatment of Individuals Infected with HBV: A US Perspective. Clin Liver Dis. 2019;23:417-32.

24. Hepatology branch of Chinese Medical Association, Infectious diseases branch of Chinese Medical Association. [The guidelines of prevention and treatment for chronic hepatitis B (2019 version)]. Zhonghua Gan Zang Bing Za Zhi. 2019;27:938-61.

25. Schwarz KB, Cloonan YK, Ling SC, et al. Children with Chronic Hepatitis B in the United States and Canada. J Pediatr. 2015;167:1287.

26. MacLachlan JH, Locarnini S, Cowie BC. Estimating the global prevalence of hepatitis B. Lancet. 2015;386:1515-7.

27. Sun YH, Lei XY, Sai YP, et al. Relationship between genotypes and clinical manifestation, pathology, and cccDNA in Chinese children with hepatitis B virus-associated glomerulonephritis. World $\mathrm{J}$ Pediatr. 2016;12:347-52.

\section{Figures}



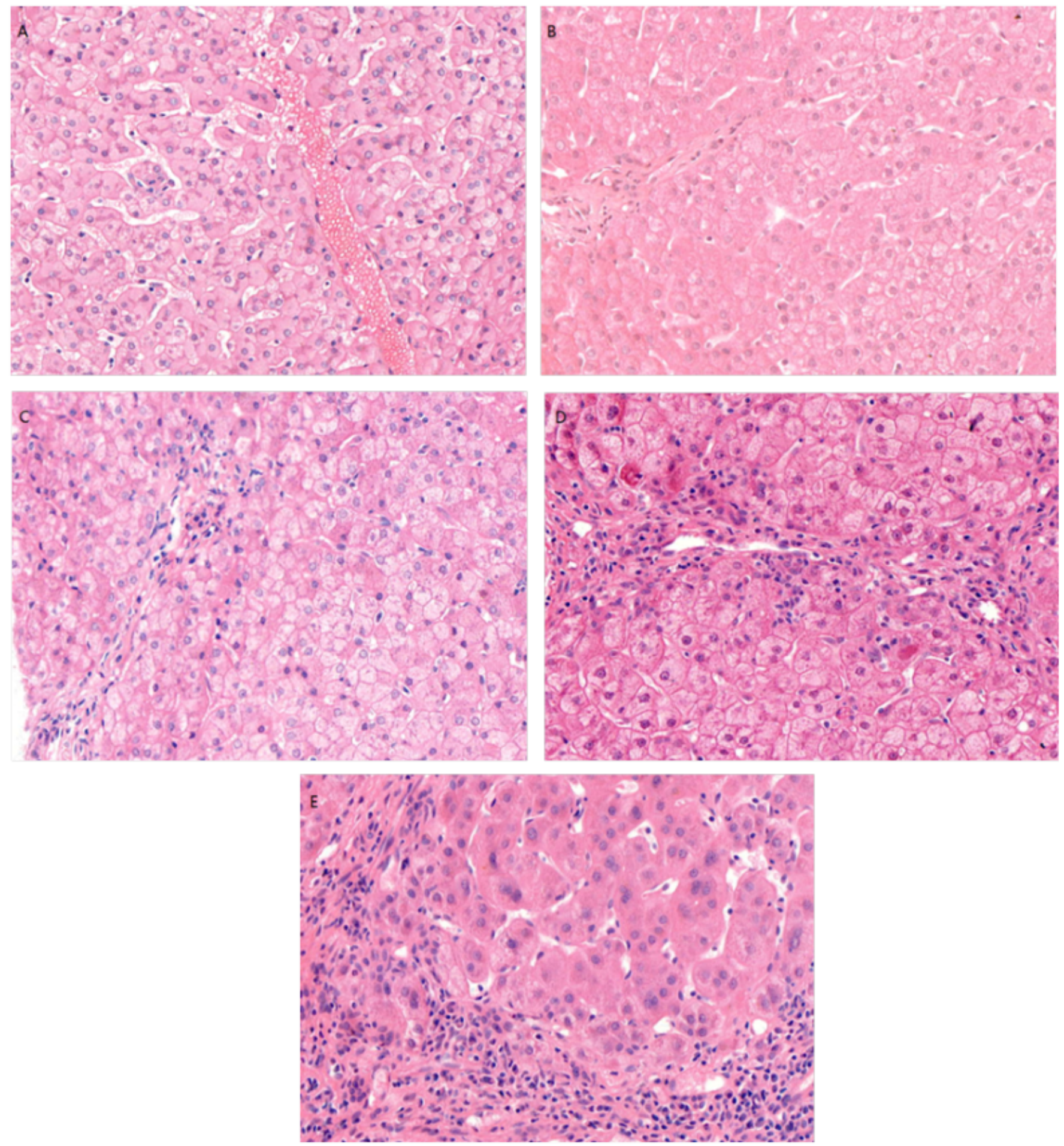

\section{Figure 1}

Liver histology of chronic hepatitis B in children (H\&E, 400x). A. G0S0, no inflammation and fibrosis. B. G1S1, mild inflammation and fibrosis. C. G2S2, moderate inflammation and fibrosis, portal inflammation with extensive interface hepatitis. D. G3S3, moderate inflammation and fibrosis, consisting of bridging fibrosis, portal lymphoid aggregate with Poulsen-type duct lesion. E. G4S4, cirrhosis with small regenerative nodules. 


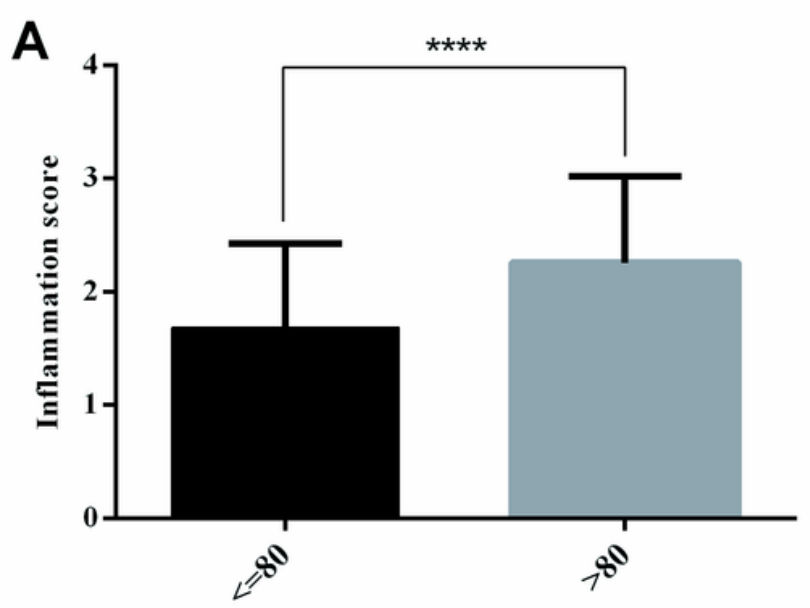

Serum ALT levels (U/L)

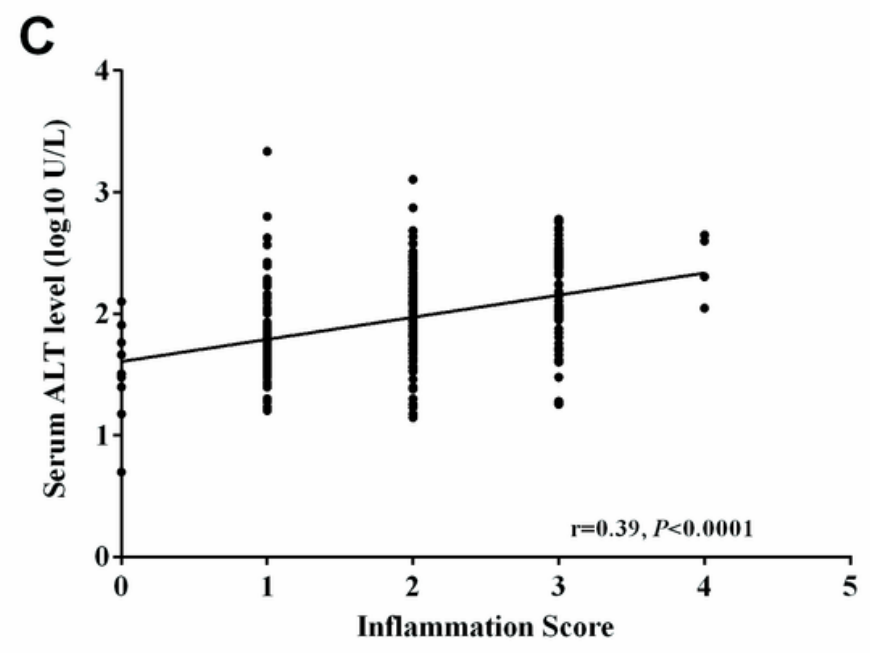

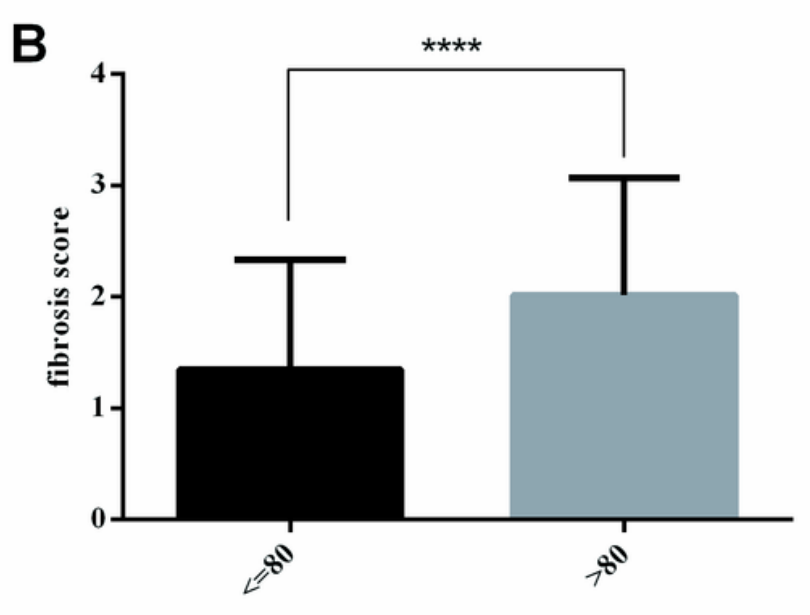

Serum ALT levels (U/L)

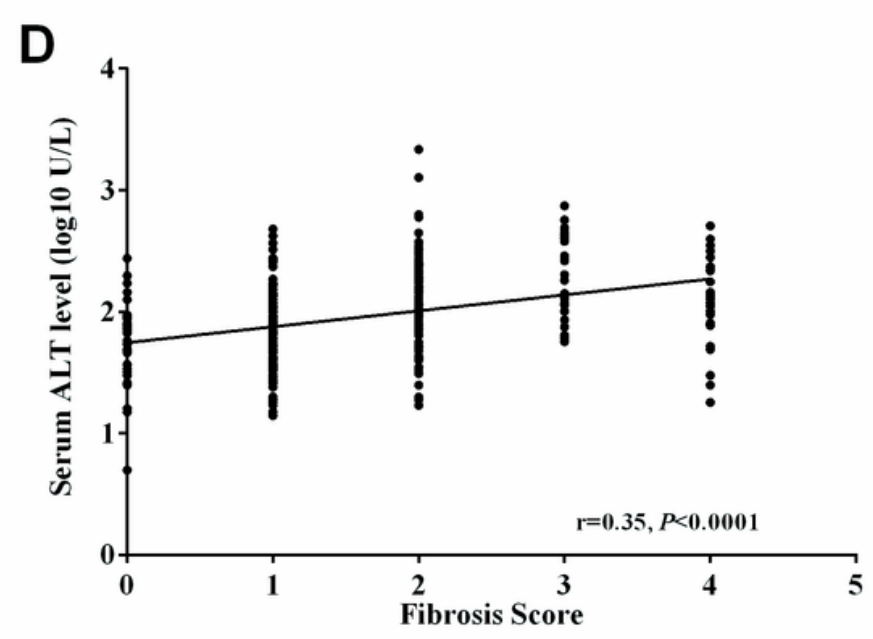

Figure 2

Association between alanine aminotransferase (ALT) and inflammation, and fibrosis.

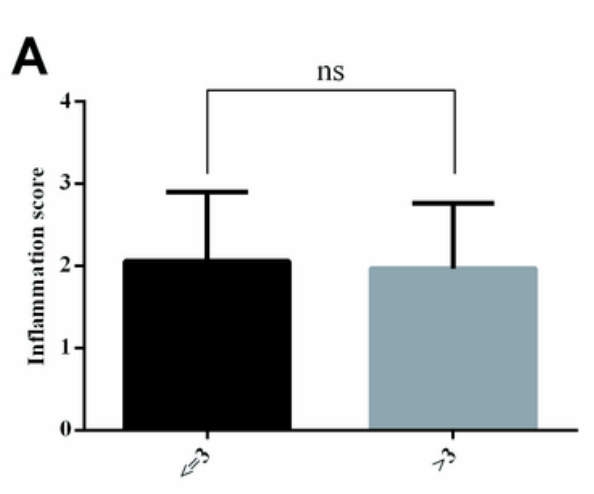

Age at biopsy (years)

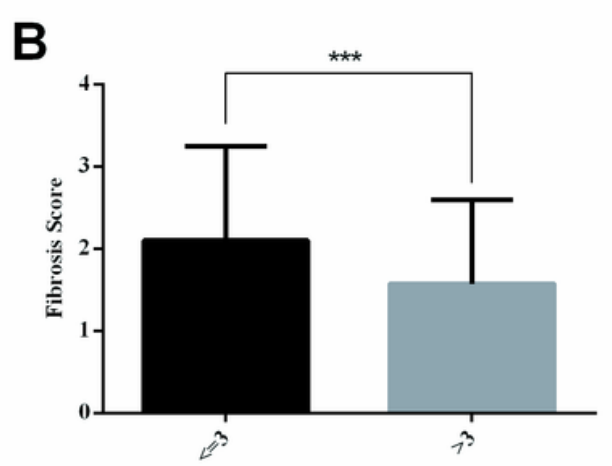

Age at biopsy (years)

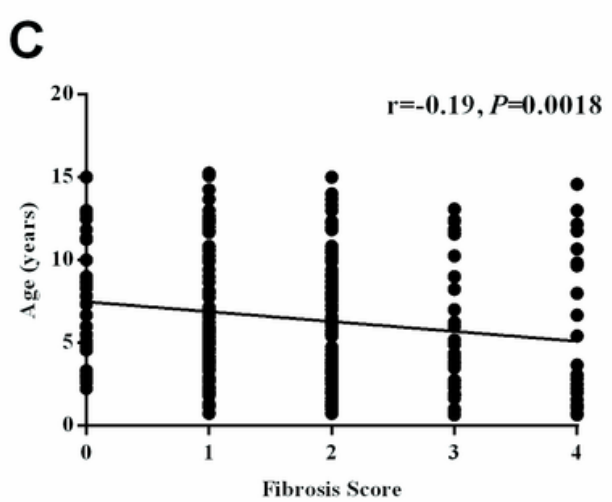

Fibrosis Score

Figure 3

Correlation between age at biopsy and histology. 